\title{
ACE2-Based Decoy Receptors for SARS Coronavirus 2
}

\author{
Wenyang Jing ${ }^{1}$ and Erik Procko ${ }^{1}$ \\ ${ }^{1}$ University of Illinois at Urbana-Champaign
}

November 3, 2020

\begin{abstract}
SARS-CoV-2 is neutralized by proteins that block receptor-binding sites on spikes that project from the viral envelope. In particular, substantial research investment has advanced monoclonal antibody therapies to the clinic where there are signs of partial efficacy in reducing viral burden and hospitalization. An alternative is to use the host entry receptor, ACE2, as a soluble decoy that broadly blocks SARS-associated coronaviruses with limited potential for viral escape. Here, we summarize efforts to engineer higher affinity variants of soluble ACE2 that rival the potency of affinity-matured antibodies. Strategies have also been used to increase the valency of ACE2 decoys for avid spike interactions and to improve pharmacokinetics via IgG fusions. Finally, the intrinsic catalytic activity of ACE2 for the turnover of the vasoconstrictor angiotensin II may directly address COVID-19 symptoms and protect against lung and cardiovascular injury, conferring dual mechanisms of action unachievable by monoclonal antibodies. Soluble ACE2 derivatives therefore have the potential to be next generation therapeutics for addressing the immediate needs of the current pandemic and possible future outbreaks.
\end{abstract}

\section{Introduction}

Not long following its emergence in China in late 2019, SARS coronavirus 2 (SARS-CoV-2) became an unprecedented public health emergency for our generation. The absence of immunity, superspreader events ${ }^{1}$ and presymptomatic ${ }^{2}$ and asymptomatic ${ }^{3,4}$ transmission have all combined to favor this respiratory virus' global spread. The virus is devastating to the elderly and other vulnerable groups with certain predisposing conditions, and elicits surprisingly heterogenous disease symptoms collectively known as COVID-19, with the most common being fever, dry cough, hypoxemia and pneumonia $a^{5,6,7,8}$, but also unusual neurological symptoms $^{9}$ and coagulopathy ${ }^{10}$. It remains unclear which aspects of disease are the result of disseminated virus infection of multiple tissues versus dysregulation of signaling pathways, including cytokine storms ${ }^{11,12}$ and aberrant angiotensin and kinin peptide processing ${ }^{13,14,15}$.

Virus entry is mediated by interactions between the viral spike, a trimeric complex of protein S, and angiotensin-converting enzyme 2 (ACE2) on a host cell membrane ${ }^{16,17,18,19,20,21,22,23}$. S is proteolytically processed as two subunits, S1 and S2, that remain noncovalently associated until ACE2 is bound by a receptor-binding domain (RBD) in the S1 subunit, triggering conformational changes that include S1 shedding, exposure of a fusion peptide at the N-terminus of S2 and fusion of the viral envelope with the host membrane $21,24,25,26,27$. Antibodies targeting multiple epitopes on S, but especially the RBD, can block ACE2 engagement or prevent membrane fusion from occurring, and numerous monoclonal antibody therapies are now in preclinical and clinical development ${ }^{28,29,30,31,32,33}$. However, coronaviruses have moderate to high mutation rates $^{34,35}$ and there is a perceived risk that drug resistant SARS-CoV-2 variants might begin circulating once antibody therapies become widely used. This is addressed by combining noncompeting monoclonal antibodies as cocktails ${ }^{36}$.

An alternative strategy is to use ACE2 itself as a soluble decoy receptor that competes for receptor-binding sites on $\mathrm{S}^{22,37,38,39}$ (Figure 1). ACE2 is an 805 amino acid (a.a.) protein that comprises a protease domain (a.a. 19-615), a collectrin-like dimerization domain (a.a. 616-729) and a single-span transmembrane domain 
(a.a. $741-765)^{16}$. The major attraction of using an entry receptor as a soluble decoy is that, in principle, the virus has limited mutational mechanisms for escape without simultaneously losing affinity for the native, membrane anchored form ${ }^{40}$. Soluble decoy receptors are used clinically for a variety of indications, although none are yet approved drugs for viruses ${ }^{41}$. Wild type, soluble ACE2 (sACE2) is an investigational drug for acute respiratory distress that has been rapidly repurposed as a SARS-CoV-2 antiviral ${ }^{37}$ and has entered a phase II COVID-19 clinical trial managed by Apeiron Biologics ${ }^{42}$. This drug candidate has become the starting point for multiple engineering efforts to solve key issues surrounding pharmacokinetics, affinity and avidity for the creation of next generation ACE2 derivatives with superior efficacy. It is these efforts that are reviewed here.

\section{Mutagenesis of ACE2 for Enhanced Affinity}

Soluble decoy receptors should ideally bind the SARS-CoV-2 spike tighter than, and out-compete, the wild type (WT) ACE2 receptor. Tight affinity is also necessary for effective virus neutralization at typical doses for biologic drugs. The importance of affinity is further emphasized when soluble decoys are considered against the therapeutic alternative of affinity-matured monoclonal antibodies, which bind S of SARS-CoV-2 with tight nanomolar to picomolar $\mathrm{K}_{\mathrm{D}}{ }^{28,29,30,33}$; by comparison, WT sACE2 has only moderate affinity (KD [?] $20 \mathrm{nM})^{16,43}$. To this end, mutagenic approaches, either targeted or through selection of diverse libraries of variants, provide a means for engineering decoy receptors with enhanced affinity.

In the first report ${ }^{44}$ that mutations within ACE2 can indeed be found that increase $\mathrm{S}$ affinity, Chan et al. performed deep mutagenesis on full length $\mathrm{ACE} 2^{43,45}$. A library of ACE2 variants was created that encoded all possible single amino acid variants at 117 sites, covering both the regions that interface with the RBD as well as the ACE2 active site. The library was then selected by fluorescence activated cell sorting (FACS) for ACE2 surface expression in human cells and tight binding to the RBD of SARS-CoV2 protein $\mathrm{S}$ (Figure 2). ACE2 residues buried at the interface with $\mathrm{S}$ tend to be more conserved, while ACE2 residues at the interface periphery or within the active site were mutationally tolerant. Mutations of ACE2 residues N90 and T92, which form an N-glycosylation motif, are universally enriched for high binding affinity (with the exception of T92S), indicating that N90 glycosylation hinders binding, at least in this experiment's cell line. Other mutations were also found dispersed across multiple sites that increase S binding, and some of these were rapidly screened as combinations of mutations in soluble ACE2 conjugated to superfolder GFP (sfGFP) ${ }^{46}$. Expression medium containing sACE2-sfGFP, without purification, can be incubated directly with cells expressing $\mathrm{S}$ and bound sACE2-sfGFP detected by flow cytometry. Ultimately, variants of sACE2 carrying four or three mutations (Figure 2C), dubbed sACE2.v2 and sACE2.v2.4, were engineered for an optimal balance of high affinity, expression and yield. Impressively, monomeric sACE2.v2, which spans ACE2 residues 19-615 that form the extracellular protease domain, outcompeted WT sACE2 fused to dimeric Fc of IgG1 and also competed effectively with anti-RBD Ig derived from Covid-19 positive patient sera. Soluble ACE2 forms a natural dimer (which we refer to as sACE2 2 ) if the construct is expanded up to ACE2 residues 730-740, which includes the dimerization/collectrin-like domain immediately C-terminal of the protease domain. Soluble ACE2 $2_{2}$ has increased apparent affinity and neutralization of authentic virus through avidity, demonstrating that each protease domain in $\mathrm{SACE} 2_{2}$ can bridge two $\mathrm{S}$ proteins. The leading variant from these efforts, dimeric sACE2 $2 . v 2.4$, had a picomolar $\mathrm{K}_{\mathrm{D}}$ for the SARS-CoV-2 RBD and achieved $50 \%$ relative infection inhibition of authentic virus at subnanomolar concentrations. Surprisingly, it showed similar neutralization efficacy against SARS-CoV-1 despite no consideration of binding to this virus during mutagenesis. This implied potential efficacy against other zoonotic coronaviruses that utilize ACE2 and may spill over to humans in the future. This was confirmed by demonstrating sACE2 $2_{2}$ v2.4 tightly binds diverse RBD sequences from SARS-related bat betacoronaviruses that use ACE2 as an entry receptor ${ }^{40}$. Furthermore, an in vitro selection of mutations within the RBD from SARS-CoV-2 failed to find S variants that lose affinity for the engineered $\mathrm{sACE} 2_{2} . \mathrm{v} 2.4$ decoy but retain binding to the wild type host ACE2 receptor, thus confirming the hypothesis that mechanisms for viruses to escape soluble decoy receptors are limited ${ }^{40}$.

In a similar deep mutagenesis experiment, Heinzelman and Romero investigated the influence on S protein 
binding of single-nucleotide variants (SNVs) in the ACE2 protease domain, evaluating nearly 4,000 amino acid substitutions ${ }^{47}$. Mutations were made using error-prone PCR, a rapid and reliable way of generating library diversity but which fails to capture multiple nucleotide changes within a single codon (unless error rates are excessively high). The library of the ACE2 protease domain was screened by yeast surface display (Figure 2B), and while most mutations decreased binding (it is, after all, easy to 'break' a protein through random mutagenesis), approximately $4 \%$ of mutations were found to increase RBD binding. While mutations in the active site tended to have little effect (with the caveat that the background ACE2 sequence used for library generation already had catalytic activity knocked out), the authors found that residues in the chloride-binding site of ACE2, which regulates its peptidase activity ${ }^{48}$ and is over $40 \AA$ from the RBD interface, do affect binding. In addition, a separate distal cluster of hydrophobic residues (L236, F588, L591, and L595) was also identified as a critical site affecting binding despite being over $30 \AA$ from the RBD interface. By interrogating SNVs, the authors were also able to statistically evaluate the clinical and epidemiological relevance of ACE2 allelic variants in the human population, and they estimated that roughly 1 in 10,000 people may have SNVs that increase spike binding and roughly 4 in 1000 having SNVs that decrease spike binding. However, these predictions will need to be confirmed by genetics studies of patient cohorts, especially since mutations in ACE2 with altered affinity for S may also have other effects that impact infection in vivo, such as expression changes in human tissues. Overall, this work highlights the importance of considering long-range effects of mutations distal from the functional binding site.

The deep mutational scans of ACE2 are based on a single round of sequence diversification and selection, whereas others have employed multiple rounds to direct the in vitro evolution of ACE2 towards exceptionally tight affinity for SARS-CoV-2 S. Higuchi et al. focused mutagenesis to ACE2 residues 18-102 and 272-409 in the protease domain, respectively denoted as PD1 and PD2, that form the RBD interface ${ }^{49}$. Binding was assayed using the method previously developed for deep mutagenesis ${ }^{43}$, with human cells expressing the ACE2 library incubated with RBD-sfGFP and sorted by FACS. Mutations within the "PD1" region were selected first, followed by mutagenesis within the "PD2" region and additional rounds of selection, although additional mutations within the PD2 region did not substantially improve affinity. The ACE2 variant identified with highest affinity, termed 3N39 with 7 substitutions (Figure 2C), bound RBD with picomolar $K_{D}$. Despite having a much higher mutational load than sACE2.v2.4, soluble 3N39-Fc fusions were soluble with minimal aggregation by size exclusion chromatography. The engineered 3N39-Fc fusion vastly outperformed the equivalent wild type ACE2-Fc fusion in virus neutralization.

The Kortemme, Hobman, and Wells groups went even further in using multiple rounds of engineering, diversification and selection to drive down the dissociation constant to the picomolar range ${ }^{50}$. Computational alanine scanning identified ACE2 residues H34, Q42 and K353 as hot spots that contribute disproportionately to the binding energy, and these residues with their neighbors were the focus for computational mutagenesis. Computationally designed ACE2 variants were purified as Fc fusions and binding to S was improved 3- to 11-fold. Four of these ACE2 variants served as parents that were then matured through yeast surface display, resulting in a further 14 -fold improvement in apparent $\mathrm{K}_{\mathrm{D}}$. Deep mutagenesis data from Chan et $\mathrm{al}^{43} \mathrm{was}_{\text {then }}$ considered, from which new ACE2 variants were designed with mutations that were not alanine scan-based hotspots and included sites outside the RBD interface that likely stabilize conformation. The final ACE2 variants had exceptionally tight affinity, with the lowest $K_{D}$ variants carrying $4-8$ substitutions (Figure $2 C$ ). It was also independently confirmed that additional increases in apparent affinity can be achieved through inclusion of the ACE2 dimerization domain for avid binding and protein stabilization. It is worth noting that in this study deliberate action was taken to inactivate ACE2 enzymatic activity. This has historically been done by mutating two critical $\mathrm{Zn}^{2+}$-coordinating residues $(\mathrm{H} 374 \mathrm{~N} \text { and } \mathrm{H} 378 \mathrm{~N})^{51}$, but because these substitutions resulted in undesirable destabilization in the variant background, an alternative mutation (H345L within the substrate-binding cavity) was adopted. While the rationale for this is the elimination of vasodilatory effects from excess ACE2 peptidase activity that may cause hypotension, it is worth considering the potential benefits of keeping enzymatic activity (discussed below).

Pangolin (Pangolin-CoV-2020) and bat (isolate RaTG13) coronaviruses, in addition to human SARS-CoV-1 and -2 , can initiate membrane fusion using not only human ACE2 but many other animal orthologs ${ }^{52}$. This 
informed the substitution of human ACE2-D30 for glutamate ${ }^{52}$, which is found in ACE2 of other species and is able to better reach K417 of the RBD for salt bridge formation ${ }^{16,17}$. In addition, inclusion of the dimerization domain together with the protease domain further facilitated the engineering of a sACE2-Ig fusion protein effective against multiple betacoronavirus strains. The discovery of the affinity-enhancing D30E mutation and benefits of including the dimerization domain overlap with independent work by others ${ }^{43,50}$. Another contributing factor was increased avidity (to be discussed further below) due to design of a tetrameric sACE2Ig configuration ${ }^{52}$. Overall, it is apparent from all these studies that a truly effective therapeutic approach utilizing decoy ACE2 receptors will likely incorporate a multifaceted strategy of affinity enrichment, avidity and Fc fusions.

One final aspect to touch upon regarding affinity enhancement through mutagenesis is the connection between binding affinity and ACE2 enzymatic activity. For example, the sACE2.v2.4 variant has reduced catalytic activity even though its 3 amino acid substitutions are not within the active site ${ }^{43}$, thus demonstrating potentially unappreciated coupling between S affinity and ACE2 catalysis. Others have observed that some mutations that knock out ACE2 activity can increase $\mathrm{S}$ binding ${ }^{53}$, although many mutations in the active site have no effect on $\mathrm{S}$ affinity ${ }^{43,47}$. In particular, we highlight again the key finding by Heinzelman and Romero that mutations to a chloride-binding motif of ACE2, which is $40 \AA$ from the RBD interface and is directly involved in regulating peptidase activity, can affect spike affinity, possibly due to conformational effects ${ }^{47}$. The binding of trimeric SARS-CoV-2 spike protein to wild type ACE2 also increases its peptidase activity 3 - to 10-fold, with the SARS-CoV-2 RBD inducing bending of the N-terminal protease subdomain toward

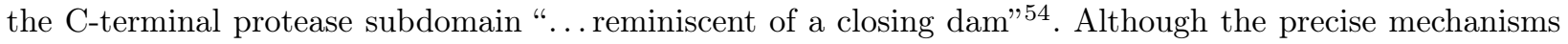
remain unclear, there are nonetheless multiple clues incriminating ACE2 conformation and dynamics on both catalytic activity and S affinity. We believe from a therapeutic perspective (to be discussed further below), maintaining catalytic activity is preferable due to the protective effects of ACE2 products on the pulmonary and cardiovascular systems.

\section{ACE2 Fusions with Immunoglobulin Fc}

Despite an ongoing clinical trial of wild type sACE2 in Europe ${ }^{42}$ and the studies reviewed above demonstrating the realization of picomolar affinity decoy receptors, it is likely that low sACE2 serum stability will limit its successful application as a therapeutic. The serum half-life of dimeric sACE2 in vivo is on the order of hours ${ }^{55,56}$, thereby requiring frequent dosing to maintain sACE2 in circulation. As a consequence, it is questionable whether sACE2 can be safely administered in an outpatient setting. Serum stability of biologic drugs is greatly increased by fusion of the therapeutic protein with the Fc region of $\operatorname{IgG}^{57}$. Serum proteins are turned over by constant internalization and lysosomal degradation, especially by endothelial cells. Internalized IgG-Fc engages the neonatal Fc receptor ( FcRn) within endosomal compartments to direct its trafficking back to the plasma membrane, thereby returning the protein to the serum (Figure 3A). The previously highlighted works describing the discovery of high affinity sACE2 variants ${ }^{43,49,50,52}$ have already extended their findings to fusions with IgG1 Fc, and we believe that sACE2-Fc fusions will be the standard going forward for the production of high-affinity decoy receptors with excellent pharmacokinetics.

Recombinant human sACE2 has been developed over nearly two decades as a therapeutic for lung inflammation and acute respiratory distress syndrome (ARDS). Its administration has been shown to be protective against cardiovascular ${ }^{58}$ and pulmonary pathologies and to treat hypoxemia in multiple animal models $^{59,60,61,62,63}$, and it has acceptable safety without causing episodes of hypotension in healthy and mechanically ventilated human subjects ${ }^{56,64}$. However, while sACE2 demonstrated the expected in vivo biochemical activity in ARDS patients, there was no statistically significant improvement in clinical outcomes in a phase 2 trial. The clinicians commented that "... lower plasma concentrations (of sACE2) over a longer duration may be more effective as a result of more sustained production of" ACE2 products $^{64}$. These findings motivated the first published report of an sACE2-Fc fusion tested in vivo (in mice), in which the serum half-life was extended to over a week with substantially higher maximum serum activity ${ }^{63}$. Recombinant sACE2-Fc mitigated hypertension following injection of the vasopressor (and ACE2 substrate) angiotensin II for up to 7 days, whereas unfused sACE2 lost function in serum within hours. The Fc fusion protein reduced 
blood pressure and cardiac and renal fibrosis in a transgenic mouse model for hypertension and albuminuria in which renin is massively overexpressed ${ }^{63}$. This work demonstrated serum longevity of sACE2 fused to IgG1-Fc as well as the potent physiological effects of ACE2 catalytic activity.

More recently with the emergence of SARS-CoV-2, multiple groups have explored IgG1-Fc fusions of sACE2 and consistently find long serum half-life without adverse toxicity in small animals ${ }^{53,38}$. The Fc region of $\operatorname{IgG}$ subclasses engage multiple Fc receptors to effect function, including not only FcRn to mediate serum stability and uptake into the lungs, but also a suite of inflammatory $\mathrm{Fc} \gamma$ receptors $(\mathrm{F} \gamma \mathrm{R})^{65}$. These receptors are expressed on a variety of cell types, especially in the immune system, and are responsible for effector functions such as antibody-dependent cellular cytotoxicity, cytokine release, antigen presentation and co-stimulation (Figure 3A). Until different Fc fusion proteins are properly assessed in controlled in vivo experiments, it is unclear whether these effector functions will aid in clearance and recovery from SARS-CoV-2 infection. Iwanaga et al have hypothesized that interactions of a sACE2-Fc biologic with inflammatory Fc $\gamma$ Rs may exacerbate inflammation and cytokine storms in COVID-19 patients ${ }^{53}$. Consequently, two leucine residues in the IgG1-Fc moiety were mutated to alanines (L234A and L235A). These so-called LALA mutations disrupt $F_{c} \gamma R$ interactions while leaving $F_{c R n}$ binding intact for high serum stability. The LALA variant of sACE2-Fc had long serum half-life, was distributed to lung tissues, and retained virus neutralization efficacy.

\section{Catalytically active or inactive sACE2?}

Most reports describing the engineering of SACE2 to enhance its affinity through targeted substitutions, or to improve pharmacokinetics through Fc fusions, have deliberately eliminated ACE2 catalytic activity ${ }^{50,53,38}$. The assumption is that administration of a catalytically active decoy receptor will adversely interact with physiology to have unacceptable toxicity. Soluble decoy receptors for other viruses have also been engineered to eliminate the normal biological activity for safety and efficacy reasons ${ }^{66}$. However, there are strong arguments that ACE2 catalytic activity, even if elevated, might be beneficial for treating COVID-19 symptoms.

In the renin-angiotensin system (RAS), a set of proteases regulates the production of angiotensin peptide hormones ${ }^{67,68}$ (Figure 3B). Renin is released by the kidneys when renal blood flow is low and it catalyzes the conversion of angiotensinogen in the blood to angiotensin I (Ang-I). Ang-I is in turn a substrate for angiotensin-converting enzyme (ACE or ACE1), which cleaves the peptide to produce the vasoconstrictor Ang-II that has a wide range of effects through the angiotensin II type 1 receptor $\left(A T_{1} R\right)$. Ang-II promotes vasoconstriction and elevated blood pressure and volume, and under conditions where Ang-II is high there can be increased inflammation, myocardial fibrosis and thrombosis. Ang-II is proteolyzed by ACE2 to produce Ang-1-7 $7^{69,70,71,72}$, which has vasodilatory properties and is cardioprotective ${ }^{73}$. Both ACE1 and ACE2 are active in lung tissue (and elsewhere) and also catalyze the conversion of kinin peptides, the consequences of which are still speculative in the context of COVID-19 ${ }^{15,74}$. Genetic studies and administration of sACE2 in animal models have demonstrated that tilting the RAS system in favor of Ang-1-7 has numerous benefits for the treatment of lung injury and inflammation ${ }^{59,60,61}$. Indeed, it has been proposed that COVID-19 symptoms may in part manifest from RAS dysregulation ${ }^{37,68}$. Ang-II levels are elevated approximately 3fold in hospitalized COVID-19 patients and are correlated with both viral load and severity of hypoxemic respiratory failure ${ }^{13}$. Catalytically active sACE2 therefore offers two potential mechanisms of action. First, it blocks receptor-binding sites on SARS-CoV-2 spikes to neutralize infection. Second, it promotes the degradation of Ang-II and production of Ang-1-7 to directly address COVID-19 symptoms. A clinical trial testing wild type sACE2 in critically ill COVID-19 patients is ongoing, but a preliminary case report offers promising signs that both mechanisms of action are at play ${ }^{42}$. This provides SACE2 a tremendous advantage over monoclonal antibodies, which are safe and effective at reducing viral load ${ }^{75}$ but do not directly address symptoms in sick patients. However, at this time no direct comparison has been made in animal models of COVID-19 to evaluate catalytically active versus inactive sACE2, alongside engineered variants with altered S affinity. The hypothesis that sACE2 will have dual mechanisms of action therefore remains unproven.

Different mutations have been introduced into sACE2 to eliminate catalytic activity, the most common being $\mathrm{H} 374 \mathrm{~N}$ and $\mathrm{H} 378 \mathrm{~N}$ to prevent coordination of an essential $\mathrm{Zn}^{2+}{ } \mathrm{ion}^{51}$. Others have used alternative mutations within the ACE2 active site ${ }^{50,53}$. As a generalization, ACE2 variants with reduced catalytic activity bind 
slightly tighter to SARS-CoV-2 S, suggesting possible conformational coupling between the catalytic and S binding sites. We are aware of only a single report ${ }^{76}$ where mutations to inactivate catalysis were associated with a small decrease in neutralization efficacy.

\section{ACE2 and Avidity}

The apparent affinity of ACE2 for S incorporates both affinity (site specific binding strength) and avidity (the total sum of all binding interactions). ACE2 is a natural dimer, and dimeric sACE2 2 is orders of magnitude more effective at neutralizing virus than the monomeric sACE2 protease domain ${ }^{43}$. The three subunits in a trimeric S spike are conformationally heterogenous, and the RBDs may be exposed for ACE2 interactions in an 'up'/'open' conformation or hidden in a 'down'/'closed' conformation ${ }^{18,77}$. The dominant conformations of prefusion S trimers are all closed or one RBD in the up state, yet there is a smaller population with additional RBDs accessible for ACE2 interactions ${ }^{78,79,80}$. There is limited evidence that dimeric ACE2 2 can bridge two $\mathrm{S}$ subunits in a single trimeric spike (i.e. intra-spike avidity $)^{81}$ and atomic structures suggest the spatial orientation of natural dimeric $\mathrm{ACE} 2_{2}$ is incompatible with binding two RBDs in a single $\mathrm{S}$ trimer. Avidity effects are therefore most likely due to dimeric ACE2 $2_{2}$ cross-linking two separate viral spikes, which is supported by cryoelectron tomography showing moderate spike density on the viral envelope suitable for bridging interactions ${ }^{78,79,80}$. However, protein engineers are not limited to the geometric architecture of natural ACE2 dimers and have at their disposal tools for creating higher multimeric forms that may sterically complement exposed RBDs on native S trimers (Figure 4).

The creation of sACE2-Fc fusions automatically forms dimeric ACE2 constructs that are superior to monomeric sACE2 $2^{50,81}$, although we do not foresee any obvious avidity improvements of Fc-mediated dimers over natural dimeric sACE2 2 . By adjusting the fusion site between sACE2 and IgG1 from the hinge to the $\mathrm{C}_{\mathrm{H}} 1$ and $\mathrm{L}_{\mathrm{H}}$ domains instead, thereby replacing the variable domains with sACE2, an assembly with four ACE2 chains is created ${ }^{52}$. This can be further expanded by fusing additional ACE2 moieties to the C-terminus of the $\mathrm{H}$ chains to create a hexavalent sACE2-IgG construct, although this arrangement compromised yield ${ }^{52}$. As expected, avid binding to spikes increases as the oligomeric assemblies get larger.

Trimeric sACE2 that spatially aligns an ACE2 moiety for interactions with each subunit of $\mathrm{S}$ in which all RBDs adopt an up conformation will have very high avidity, and has been explored by two groups ${ }^{82,83}$. The ACE2 protease domain has been fused to trimerization motifs: the C-terminal domain of T4 fibritin (or foldon) and a three helix bundle. The sACE2 trimers can simultaneously bind multiple RBDs in a spike with low picomolar apparent $\mathrm{K}_{\mathrm{D}}$. The sACE2-foldon trimer was found to have slightly better inhibitory activity against pseudotyped virus. Affinity-enhancing mutations have also been combined with trimeric sACE2-foldon ${ }^{83}$.

Lastly, avidity has been increased by fusing an anti-S antibody to sACE2 to create a hybrid, biparatopic ACE2-IgG fusion ${ }^{84}$. While this protein still contains two ACE2 moieties like other sACE2-IgG fusions, it is distinct in that avidity is also increased by combining bivalent ACE2 binding with a neutralizing antibody that recognizes a non-competing epitope on the spike N-terminal domain. The antibody was selected by yeast display from SARS-CoV-2 survivors. The biparatopic ACE2-IgG hybrid has greatly improved neutralization potency against pseudotyped and authentic virus. Whether this fusion construct compromises serum stability remains to be determined.

\section{Vesicle-Based ACE2 Nanoparticle Decoys}

While this review has focused on soluble ACE2 receptors and their associated Ig-fusions, work has also been done utilizing membrane-anchored ACE2. These decoys are based on cell-derived vesicles or fabricated nanoparticles derived from cells expressing membrane-embedded ACE2 ${ }^{85,86,87}$. Extracellular vesicles produced from cells over-expressing ACE2 together with TMPRSS2 - which is required for membrane fusion following ACE2/S binding ${ }^{21}$ — were more effective at neutralizing pseudotyped viruses than purified sACE2, perhaps because of inbuilt avidity from high density receptor display ${ }^{86}$. TMPRSS2 was required for optimum activity, and it is possible bound viruses fused with the vesicles, effectively creating irreversible neutralization. Vesicles have been further functionalized by the use of membranes from monocytes that 
naturally express cytokine receptors, such that both virus and cytokines are sequestered on the nanoparticle surface $^{87}$. Inhibited cytokine signaling might ameliorate potentially life-threatening inflammatory responses associated with severe COVID-19. Cytokine neutralization activity was confirmed in vivo by suppressed inflammation in a lipopolysaccharide-induced lung injury model in mice. While vesicle-based nanoparticles are scientifically exciting and show efficacy for virus and cytokine neutralization, they will almost certainly suffer a tortuous regulatory pathway to the clinic due to composition heterogeneity and safety concerns.

\section{Conclusion}

Emergency Use Authorization from the U.S. Food and Drug Administration is currently being sought for two monoclonal antibody therapies for COVID-19, with others under development. It has been conclusively demonstrated that soluble ACE2 decoys can be engineered to rival the affinities of monoclonal antibodies, and provided IgG fusions confer desirable pharmacokinetics in humans, there is no reason to expect soluble ACE2 decoys cannot be as effective as monoclonals in reducing viral load in patients. Anti-SARS-CoV-2 monoclonals have received a preferential regulatory environment for clinical advancement due to an in-depth understanding of antibody biochemistry, pharmacokinetics and general toxicology. Decoy receptors will always come with additional risks related to immunogenicity and safety, and the outstanding question for the community is whether they provide truly unique benefits that outweigh those risks. The advantages of soluble ACE2 are its breadth against SARS-associated coronaviruses and its potential for alleviating COVID-19 symptoms through intrinsic enzymatic activity. We believe these advantages justify continued efforts towards advancing these proteins to the clinic.

\section{Acknowledgements}

E.P. is supported by NIH award R01AI129719.

\section{Conflicts of Interest}

The University of Illinois has filed a provisional patent for engineered decoy receptors with E.P. as an inventor. E.P. is a co-founder of Orthogonal Biologics, Inc.

\section{References}

1. Adam DC, Wu P, Wong JY, Lau EHY, Tsang TK, Cauchemez S, Leung GM, Cowling BJ. Clustering and superspreading potential of SARS-CoV-2 infections in Hong Kong. Nat Med 2020;

2. He X, Lau EHY, Wu P, Deng X, Wang J, Hao X, Lau YC, Wong JY, Guan Y, Tan X, et al. Temporal dynamics in viral shedding and transmissibility of COVID-19. Nat Med 2020;26:672-675.

3. Bai Y, Yao L, Wei T, Tian F, Jin D-Y, Chen L, Wang M. Presumed Asymptomatic Carrier Transmission of COVID-19. JAMA 2020;323:1406-1407.

4. Long QX, Tang XJ, Shi QL, Li Q, Deng HJ, Yuan J, Hu JL, Xu W, Zhang Y, Lv FJ, et al. Clinical and immunological assessment of asymptomatic SARS-CoV-2 infections. Nat Med 2020;26:1200-1204.

5. Zhu N, Zhang D, Wang W, Li X, Yang B, Song J, Zhao X, Huang B, Shi W, Lu R, et al. A Novel Coronavirus from Patients with Pneumonia in China, 2019. N Engl J Med 2020;382:727-733.

6. Huang C, Wang Y, Li X, Ren L, Zhao J, Hu Y, Zhang L, Fan G, Xu J, Gu X, et al. Clinical features of patients infected with 2019 novel coronavirus in Wuhan, China. Lancet 2020;395:497-506.

7. Zheng Z, Peng F, Xu B, Zhao J, Liu H, Peng J, Li Q, Jiang C, Zhou Y, Liu S, et al. Risk factors of critical \& mortal COVID-19 cases: A systematic literature review and meta-analysis. J Infect 2020;81:e16-e25.

8. Wang W, Tang J, Wei F. Updated understanding of the outbreak of 2019 novel coronavirus (2019-nCoV) in Wuhan, China. J Med Virol 2020;92:441-447.

9. Ellul MA, Benjamin L, Singh B, Lant S, Michael BD, Easton A, Kneen R, Defres S, Sejvar J, Solomon T. Neurological associations of COVID-19. Lancet Neurol 2020;19:767-783. 
10. Becker RC. COVID-19 update: Covid-19-associated coagulopathy. J Thromb Thrombolysis 2020;50:5467.

11. Soy M, Keser G, Atagündüz P, Tabak F, Atagündüz I, Kayhan S. Cytokine storm in COVID-19: pathogenesis and overview of anti-inflammatory agents used in treatment. Clin Rheumatol 2020;39:20852094.

12. Song P, Li W, Xie J, Hou Y, You C. Cytokine storm induced by SARS-CoV-2. Clin Chim Acta 2020;509:280-287.

13. Liu Y, Yang Y, Zhang C, Huang F, Wang F, Yuan J, Wang Z, Li J, Li J, Feng C, et al. Clinical and biochemical indexes from 2019-nCoV infected patients linked. Sci China Life Sci 2020;63:364-374.

14. Guo J, Huang Z, Lin L, Lv J. Coronavirus Disease 2019 (COVID-19) and Cardiovascular Disease: A Viewpoint on the Potential Influence of Angiotensin-Converting Enzyme Inhibitors/Angiotensin Receptor Blockers on Onset and Severity of Severe Acute Respiratory Syndrome Coronavirus 2 Infection. J Am Heart Assoc 2020;9:e016219.

15. Roche JA, Roche R. A hypothesized role for dysregulated bradykinin signaling in COVID-19 respiratory complications. FASEB J 2020;34:7265-7269.

16. Yan R, Zhang Y, Li Y, Xia L, Guo Y, Zhou Q. Structural basis for the recognition of SARS-CoV-2 by full-length human ACE2. Science 2020;367:1444-1448.

17. Lan J, Ge J, Yu J, Shan S, Zhou H, Fan S, Zhang Q, Shi X, Wang Q, Zhang L, et al. Structure of the SARS-CoV-2 spike receptor-binding domain bound to the ACE2 receptor. Nature 2020;581:215-220.

18. Wrapp D, Wang N, Corbett KS, Goldsmith JA, Hsieh CL, Abiona O, Graham BS, McLellan JS. Cryo-EM structure of the 2019-nCoV spike in the prefusion conformation. Science 2020;367:1260-1263.

19. Zhou P, Yang X Lou, Wang XG, Hu B, Zhang L, Zhang W, Si HR, Zhu Y, Li B, Huang CL, et al. A pneumonia outbreak associated with a new coronavirus of probable bat origin. Nature 2020;579:270-273.

20. Wan Y, Shang J, Graham R, Baric RS, Li F. Receptor Recognition by the Novel Coronavirus from Wuhan: an Analysis Based on Decade-Long Structural Studies of SARS Coronavirus. J Virol 2020;94:1-9.

21. Hoffmann M, Kleine-Weber H, Schroeder S, Krüger N, Herrler T, Erichsen S, Schiergens TS, Herrler G, Wu NH, Nitsche A, et al. SARS-CoV-2 Cell Entry Depends on ACE2 and TMPRSS2 and Is Blocked by a Clinically Proven Protease Inhibitor. Cell 2020;181:271-280.e8.

22. Li W, Moore MJ, Vasilieva N, Sui J, Wong SK, Berne MA, Somasundaran M, Sullivan JL, Luzuriaga K, Greenough TC, et al. Angiotensin-converting enzyme 2 is a functional receptor for the SARS coronavirus. Nature 2003;426:450-454.

23. Letko M, Marzi A, Munster V. Functional assessment of cell entry and receptor usage for SARS-CoV-2 and other lineage B betacoronaviruses. Nat Microbiol 2020;5:562-569.

24. Tortorici MA, Veesler D. Chapter Four - Structural insights into coronavirus entry. In: Rey FA, editor. Advances in Virus Research. Academic Press; 2019. p 93-116.

25. Walls AC, Park YJ, Tortorici MA, Wall A, McGuire AT, Veesler D. Structure, Function, and Antigenicity of the SARS-CoV-2 Spike Glycoprotein. Cell 2020;181:281-292.e6.

26. Mille JK, Whittaker GR. Host cell entry of Middle East respiratory syndrome coronavirus after two-step, furin-mediated activation of the spike protein. Proc Natl Acad Sci U S A 2014;111:15214-15219.

27. Walls AC, Tortorici MA, Snijder J, Xiong X, Bosch BJ, Rey FA, Veesler D. Tectonic conformational changes of a coronavirus spike glycoprotein promote membrane fusion. Proc Natl Acad Sci U S A 2017;114:11157-11162. 
28. Hansen J, Baum A, Pascal KE, Russo V, Giordano S, Wloga E, Fulton BO, Yan Y, Koon K, Patel K, et al. Studies in humanized mice and convalescent humans yield a SARS-CoV-2 antibody cocktail. Science 2020;369:1010-1014.

29. Brouwer PJM, Caniels TG, van der Straten K, Snitselaar JL, Aldon Y, Bangaru S, Torres JL, Okba NMA, Claireaux M, Kerster G, et al. Potent neutralizing antibodies from COVID-19 patients define multiple targets of vulnerability. Science 2020;369:643-650.

30. Wec AZ, Wrapp D, Herbert AS, Maurer DP, Haslwanter D, Sakharkar M, Jangra RK, Eugenia Dieterle M, Lilov A, Huang D, et al. Broad neutralization of SARS-related viruses by human monoclonal antibodies. Science 2020;369:731-736.

31. Wang C, Li W, Drabek D, Okba NMA, van Haperen R, Osterhaus ADME, van Kuppeveld FJM, Haagmans BL, Grosveld F, Bosch BJ. A human monoclonal antibody blocking SARS-CoV-2 infection. Nat Commun 2020;11:1-6.

32. Wu Y, Wang F, Shen C, Peng W, Li D, Zhao C, Li Z, Li S, Bi Y, Yang Y, et al. A noncompeting pair of human neutralizing antibodies block COVID-19 virus binding to its receptor ACE2. Science 2020;368:12741278 .

33. Rogers TF, Zhao F, Huang D, Beutler N, Burns A, He W, Limbo O, Smith C, Song G, Woehl J, et al. Isolation of potent SARS-CoV-2 neutralizing antibodies and protection from disease in a small animal model. Science 2020;369:956- 963.

34. Pyrc K, Dijkman R, Deng L, Jebbink MF, Ross HA, Berkhout B, van der Hoek L. Mosaic Structure of Human Coronavirus NL63, One Thousand Years of Evolution. J Mol Biol 2006;364:964-973.

35. Su S, Wong G, Shi W, Liu J, Lai ACK, Zhou J, Liu W, Bi Y, Gao GF. Epidemiology, Genetic Recombination, and Pathogenesis of Coronaviruses. Trends Microbiol 2016;24:490-502.

36. Baum A, Fulton BO, Wloga E, Copin R, Pascal KE, Russo V, Giordano S, Lanza K, Negron N, Ni M, et al. Antibody cocktail to SARS-CoV-2 spike protein prevents rapid mutational escape seen with individual antibodies. Science 2020;369:1014- 1018 .

37. Monteil V, Kwon H, Prado P, Hagelkrüys A, Wimmer RA, Stahl M, Leopoldi A, Garreta E, Hurtado del Pozo C, Prosper F, et al. Inhibition of SARS-CoV-2 Infections in Engineered Human Tissues Using Clinical-Grade Soluble Human ACE2. Cell 2020;181:905-913.e7.

38. Lei C, Qian K, Li T, Zhang S, Fu W, Ding M, Hu S. Neutralization of SARS-CoV-2 spike pseudotyped virus by recombinant ACE2-Ig. Nat Commun 2020;11:1-5.

39. Hofmann H, Geier M, Marzi A, Krumbiegel M, Peipp M, Fey GH, Gramberg T, Pöhlmann S. Susceptibility to SARS coronavirus S protein-driven infection correlates with expression of angiotensin converting enzyme 2 and infection can be blocked by soluble receptor. Biochem Biophys Res Commun 2004;319:12161221.

40. Chan KK, Tan TJC, Narayanan KK, Procko E. An engineered decoy receptor for SARS-CoV-2 broadly binds protein S sequence variants. bioRxiv 2020;10.18.344622.

41. Usmani SS, Bedi G, Samuel JS, Singh S, Kalra S, Kumar P, Ahuja AA, Sharma M, Gautam A, Raghava GPS. THPdb: Database of FDA-approved peptide and protein therapeutics. PLoS One 2017;12:1-12.

42. Zoufaly A, Poglitsch M, Aberle JH, Hoepler W, Seitz T, Traugott M, Grieb A, Pawelka E, Laferl H, Wenisch C, et al. Human recombinant soluble ACE2 in severe COVID-19. Lancet Respir Med 2020;2600:1-5.

43. Chan KK, Dorosky D, Sharma P, Abbasi SA, Dye JM, Kranz DM, Herbert AS, Procko E. Engineering human ACE2 to optimize binding to the spike protein of SARS coronavirus 2. Science 2020;369:1261-1265. 
44. Procko E. The sequence of human ACE2 is suboptimal for binding the $\mathrm{S}$ spike protein of SARS coronavirus 2. bioRxiv 2020;03.16.994236.

45. Procko E. Deep mutagenesis in the study of COVID-19: a technical overview for the proteomics community. Expert Rev Proteomics 2020;1-6.

46. Pédelacq JD, Cabantous S, Tran T, Terwilliger TC, Waldo GS. Engineering and characterization of a superfolder green fluorescent protein. Nat Biotechnol 2006;24:79-88.

47. Heinzelman P, Romero PA. Discovery of human ACE2 variants with altered recognition by the SARSCoV-2 spike protein. bioRxiv 2020;09.17.301861.

48. Rushworth CA, Guy JL, Turner AJ. Residues affecting the chloride regulation and substrate selectivity of the angiotensin-converting enzymes (ACE and ACE2) identified by site-directed mutagenesis. FEBS J 2008;275:6033-6042.

49. Higuchi Y, Suzuki T, Arimori T, Ikemura N, Kirita Y, Ohgitani E, Mazda O, Motooka D, Nakamura S, Matsuura Y, et al. High affinity modified ACE2 receptors prevent SARS-CoV-2 infection. bioRxiv 2020;09.16.299891.

50. Glasgow A, Glasgow J, Limonta D, Solomon P, Lui I, Zhang Y, Nix MA, Rettko NJ, Zha S, Yamin R, et al. Engineered ACE2 receptor traps potently neutralize SARS-CoV-2. Proc Natl Acad Sci 2020;202016093.

51. Moore MJ, Dorfman T, Li W, Wong SK, Li Y, Kuhn JH, Coderre J, Vasilieva N, Han Z, Greenough TC, et al. Retroviruses Pseudotyped with the Severe Acute Respiratory Syndrome Coronavirus Spike Protein Efficiently Infect Cells Expressing Angiotensin-Converting Enzyme 2. J Virol 2004;78:10628-10635.

52. Li Y, Wang H, Tang X, Fang S, Ma D, Du C, Wang Y, Pan H, Yao W, Zhang R, et al. SARS-CoV-2 and three related coronaviruses utilize multiple ACE2 orthologs and are potently blocked by an improved ACE2-Ig. J Virol 2020;

53. Iwanaga N, Cooper L, Rong L, Beddingfield B, Crabtree J, Tripp A, Qin X, Kolls JK. Novel ACE2-IgG1 fusions with improved in vitro and in vivo activity against SARS-CoV2. bioRxiv 2020;06.15.152157.

54. Lu J, Sun PD. High affinity binding of SARS-CoV-2 spike protein enhances ACE2 carboxypeptidase activity. J Biol Chem 2020;RA120:015303.

55. Wysocki J, Ye M, Rodriguez E, González-Pacheco FR, Barrios C, Evora K, Schuster M, Loibner H, Brosnihan KB, Ferrario CM, et al. Targeting the degradation of angiotensin II with recombinant angiotensinconverting enzyme 2: Prevention of angiotensin II-dependent hypertension. Hypertension 2010;55:90-98.

56. Haschke M, Schuster M, Poglitsch M, Loibner H, Salzberg M, Bruggisser M, Penninger J, Krähenbühl S. Pharmacokinetics and pharmacodynamics of recombinant human angiotensin-converting enzyme 2 in healthy human subjects. Clin Pharmacokinet 2013;52:783-792.

57. Strohl WR. Fusion Proteins for Half-Life Extension of Biologics as a Strategy to Make Biobetters. BioDrugs 2015;29:215-239.

58. Crackower MA, Sarao R, Oliveira-dos-Santos AJ, Da Costa J, Zhang L. Angiotensin-converting enzyme 2 is an essential regulator of heart function. Nature 2002;417:822-828.

59. Imai Y, Kuba K, Rao S, Huan Y, Guo F, Guan B, Yang P, Sarao R, Wada T, Leong-Poi H, et al. Angiotensin-converting enzyme 2 protects from severe acute lung failure. Nature 2005;436:112-116.

60. Zou Z, Yan Y, Shu Y, Gao R, Sun Y, Li X, Ju X, Liang Z, Liu Q, Zhao Y, et al. Angiotensin-converting enzyme 2 protects from lethal avian influenza A H5N1 infections. Nat Commun 2014;5:1-7.

61. Treml B, Neu N, Kleinsasser A, Gritsch C, Finsterwalder T, Geiger R, Schuster M, Janzek E, Loibner $\mathrm{H}$, Penninger J, et al. Recombinant angiotensin-converting enzyme 2 improves pulmonary blood flow and oxygenation in lipopolysaccharide-induced lung injury in piglets. Crit Care Med 2010;38. 
62. Johnson JA, West J, Maynard KB, Hemnes AR. ACE2 improves right ventricular function in a pressure overload model. PLoS One 2011;6.

63. Liu P, Wysocki J, Souma T, Ye M, Ramirez V, Zhou B, Wilsbacher LD, Quaggin SE, Batlle D, Jin J. Novel ACE2-Fc chimeric fusion provides long-lasting hypertension control and organ protection in mouse models of systemic renin angiotensin system activation. Kidney Int 2018;94:114-125.

64. Khan A, Benthin C, Zeno B, Albertson TE, Boyd J, Christie JD, Hall R, Poirier G, Ronco JJ, Tidswell $\mathrm{M}$, et al. A pilot clinical trial of recombinant human angiotensin-converting enzyme 2 in acute respiratory distress syndrome. Crit Care 2017;21:1-9.

65. Saunders KO. Conceptual Approaches to Modulating Antibody Effector Functions and Circulation HalfLife. Front Immunol 2019;10:1296.

66. Park J, Gill KS, Aghajani AA, Heredia JD, Choi H, Oberstein A, Procko E. Engineered receptors for human cytomegalovirus that are orthogonal to normal human biology. PLOS Pathog 2020;16:e1008647.

67. Kuba K, Imai Y, Ohto-Nakanishi T, Penninger JM. Trilogy of ACE2: A peptidase in the renin-angiotensin system, a SARS receptor, and a partner for amino acid transporters. Pharmacol Ther 2010;128:119-128.

68. Gheblawi M, Wang K, Viveiros A, Nguyen Q, Zhong J-C, Turner AJ, Raizada MK, Grant MB, Oudit GY. Angiotensin-Converting Enzyme 2: SARS-CoV-2 Receptor and Regulator of the Renin-Angiotensin System: Celebrating the 20th Anniversary of the Discovery of ACE2. Circ Res 2020;126:1456-1474.

69. Tipnis SR, Hooper NM, Hyde R, Karran E, Christie G, Turner AJ. A human homolog of angiotensinconverting enzyme. Cloning and functional expression as a captopril-insensitive carboxypeptidase. J Biol Chem 2000;275:33238-33243.

70. Turner AJ, Tipnis SR, Guy JL, Rice G, Hooper NM. ACEH/ACE2 is a novel mammalian metallocarboxypeptidase and a homologue of angiotensin-converting enzyme insensitive to ACE inhibitors. Can J Physiol Pharmacol 2002;80:346-353.

71. Vickers C, Hales P, Kaushik V, Dick L, Gavin J, Tang J, Godbout K, Parsons T, Baronas E, Hsieh F, et al. Hydrolysis of biological peptides by human angiotensin-converting enzyme-related carboxypeptidase. J Biol Chem 2002;277:14838-14843.

72. Donoghue M, Hsieh F, Baronas E, Godbout K, Gosselin M, Stagliano N, Donovan M, Woolf B, Robison $\mathrm{K}$, Jeyaseelan R, et al. A novel angiotensin-converting enzyme-related carboxypeptidase (ACE2) converts angiotensin I to angiotensin 1-9. Circ Res 2000;87:E1-9.

73. Dasgupta C, Zhang L. Angiotensin II receptors and drug discovery in cardiovascular disease. Drug Discov Today 2011;16:22-34.

74. Garvin MR, Alvarez C, Miller JI, Prates ET, Walker AM, Amos BK, Mast AE, Justice A, Aronow B, Jacobson D. A mechanistic model and therapeutic interventions for COVID-19 involving a RAS-mediated bradykinin storm. Elife 2020;9:e59177.

75. Chen P, Nirula A, Heller B, Gottlieb RL, Boscia J, Morris J, Huhn G, Cardona J, Mocherla B, Stosor V, et al. SARS-CoV-2 Neutralizing Antibody LY-CoV555 in Outpatients with Covid-19. N Engl J Med 2020;

76. Liu P, Xie X, Gao L, Jin J. Designed Variants of Recombinant ACE2-Fc that Decouple Anti-SARS-CoV-2 Activities from Unwanted Cardiovascular Effects. bioRxiv 2020;08.13.248351.

77. Huang Y, Yang C, Xu X, Xu W, Liu S. Structural and functional properties of SARS-CoV-2 spike protein: potential antivirus drug development for COVID-19. Acta Pharmacol Sin 2020;41:1141-1149.

78. Ke Z, Oton J, Qu K, Cortese M, Zila V, McKeane L, Nakane T, Zivanov J, Neufeldt CJ, Cerikan B, et al. Structures and distributions of SARS-CoV-2 spike proteins on intact virions. Nature 2020; 
79. Turoňová B, Sikora M, Schürmann C, Hagen WJH, Welsch S, Blanc FEC, von Bülow S, Gecht M, Bagola K, Hörner C, et al. In situ structural analysis of SARS-CoV-2 spike reveals flexibility mediated by three hinges. Science 2020;370:203- 208.

80. Yao H, Song Y, Chen Y, Wu N, Xu J, Sun C, Zhang J, Weng T, Zhang Z, Wu Z, et al. Molecular Architecture of the SARS-CoV-2 Virus. Cell 2020;183:730-738.e13.

81. Lui I, Zhou XX, Lim SA, Elledge SK, Solomon P, Rettko NJ, Zha BS, Kirkemo LL, Gramespacher JA, Liu J, et al. Trimeric SARS-CoV-2 Spike interacts with dimeric ACE2 with limited intra-Spike avidity. bioRxiv 2020;05.21.109157.

82. Guo L, Bi W, Wang X, Xu W, Yan R, Zhang Y, Zhao K, Li Y, Zhang M, Bao X, et al. Engineered Trimeric ACE2 Binds and Locks "Three-up" Spike Protein to Potently Inhibit SARS-CoVs and Mutants. bioRxiv 2020;2020.08.31.274704.

83. Xiao T, Lu J, Zhang J, Johnson RI, McKay LGA, Storm N, Lavine CL, Peng H, Cai Y, Rits-Volloch $\mathrm{S}$, et al. A trimeric human angiotensin-converting enzyme 2 as an anti-SARS-CoV-2 agent in vitro. bioRxiv 2020;09.18.301952.

84. Miao X, Luo Y, Huang X, Lee SMY, Yuan Z, Tang Y, Chen L, Wang C, Wu F, Xu Y, et al. A novel biparatopic hybrid antibody-ACE2 fusion that blocks SARS-CoV-2 infection: implications for therapy. MAbs $2020 ; 12$.

85. Wang C, Wang S, 1\# C, Zhao J, Han S, Zhao G, Kang J, Liu Y, Wang L, Wang X, et al. Membrane Nanoparticles Derived from ACE2-rich Cells Block SARS-CoV-2 Infection. bioRxiv 2020;08.12.247338.

86. Cocozza F, Piovesana E, Névo N, Lahaye X, Buchrieser J, Schwartz O, Manel N, Tkach M, Théry C, Martin-Jaular L. Extracellular vesicles containing ACE2 efficiently prevent infection by SARS-CoV-2 Spike protein-containing virus. bioRxiv 2020;07.08.193672.

87. Rao L, Xia S, Xu W, Tian R, Yu G, Gu C, Pan P, Meng Q-F, Cai X, Qu D, et al. Decoy nanoparticles protect against COVID-19 by concurrently adsorbing viruses and inflammatory cytokines. Proc Natl Acad Sci 2020;2:202014352.

Figure 1. Soluble ACE2 neutralizes SARS-CoV-2 infection.Soluble ACE2 (violet) competes with native, membrane-anchored ACE2 receptors (blue) for binding sites on viral spikes (green).

Figure 2. Selection strategies for enhancing the affinity of ACE2 for the RBD of SARS-CoV2. (A) In human cell selections of ACE2 variants, surface expression of full-length ACE2 with N-terminal epitope tags was detected via fluorescent anti-tag antibodies. Cells expressing ACE2 clones with high binding to RBD-sfGFP were collected by FACS.(B) In FACS selections of yeast, the protease domain of ACE2 was expressed as a fusion to Aga2p for display on the yeast surface. Displayed protein was detected via a Cterminal fusion to GFP or immunostaining of an epitope tag in the connecting linker. Bound RBD was labeled via biotin or an epitope tag for fluorescence detection.(C) Structure (PDB 6M17) of RBD (green) bound to ACE2 (pale blue). Common mutations in high affinity engineered decoys are indicated in parentheses. (D) Mutational landscape showing how single amino acid substitutions in ACE2 increase (blue) or decrease (red) binding to RBD. Data are from Chan et $\mathrm{al}^{43}$.

Figure 3. Common modifications to soluble ACE2: fusions with IgG-Fc and elimination of catalytic activity. (A) The Fc region of IgG can be fused to the extracellular domains of ACE2. The Fc moiety is recognized by inflammatory FcrRs on various cell types for immune effector functions. IgG-Fc also binds FcRn within endosomal compartments following pinocytosis by endothelial and epithelial cells to mediate recycling or transcytosis, impacting biodistribution and serum stability. (B) The conversion of angiotensin peptide hormones by renin, ACE1 and ACE2.

Figure 4. Strategies to increase $\mathbf{S A C E 2}{ }^{*} \mathrm{~S}$ avidity. $\mathrm{S}$ of SARS-CoV-2 binds the monomeric ACE2 protease domain (a.a. 19-615). A longer version of sACE2 (a.a. 19-732/740) forms a stable dimer that 
avidly binds virus. Higher levels of multimerization can be accomplished by various N- or C-terminal fusions to IgG heavy and light chains. Trimeric sACE2 proteins have also been constructed to complement trimeric $\mathrm{S}$ spikes. Finally, ACE2 has been fused to an anti-SARS-CoV-2 monoclonal to create an avid biparatopic antibody hybrid.

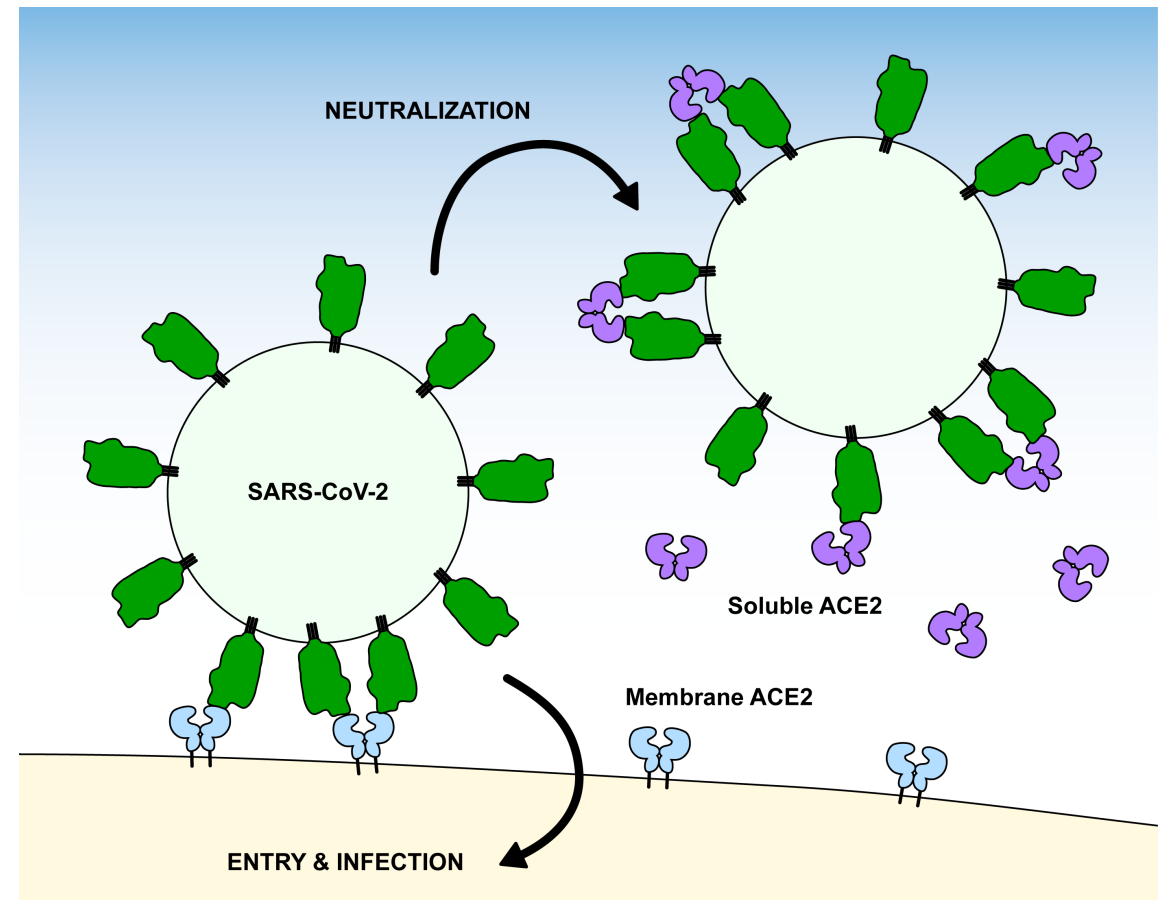

A

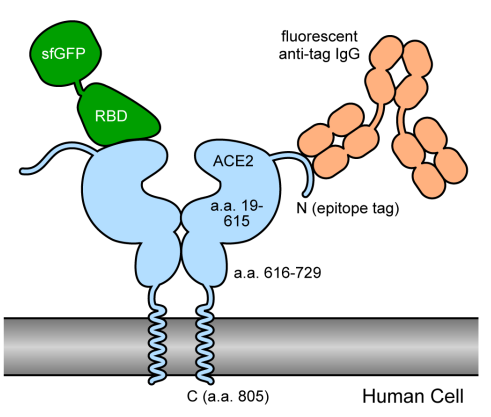

C

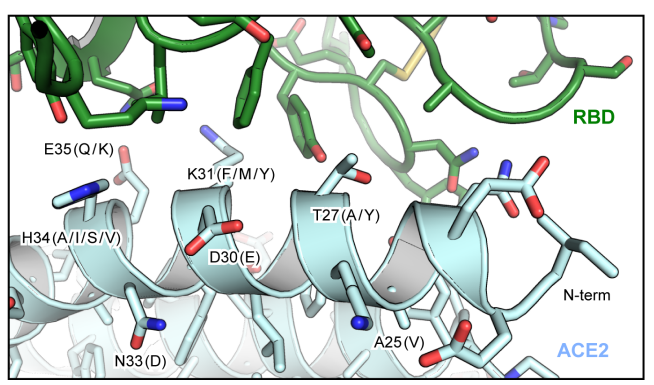

B

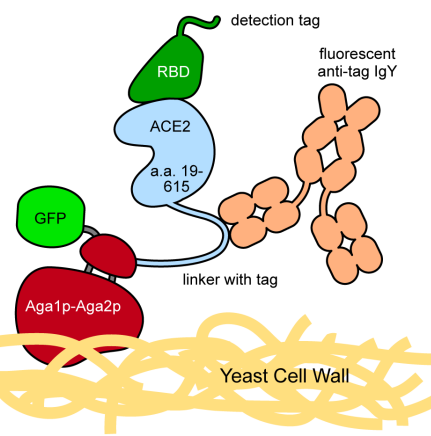

$\mathrm{D}$

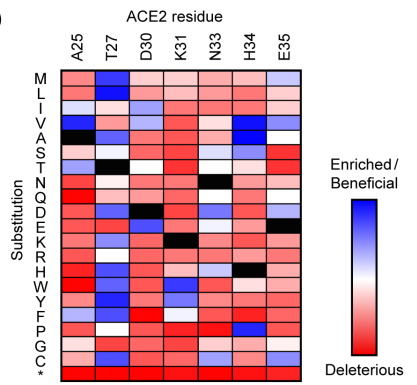




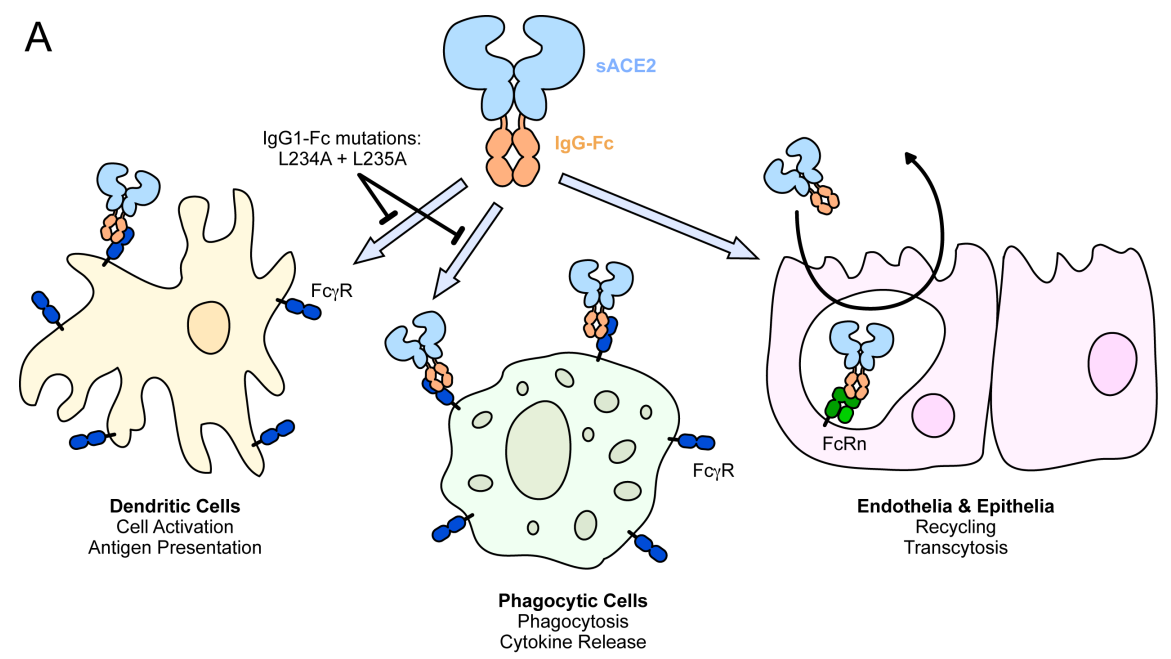

B
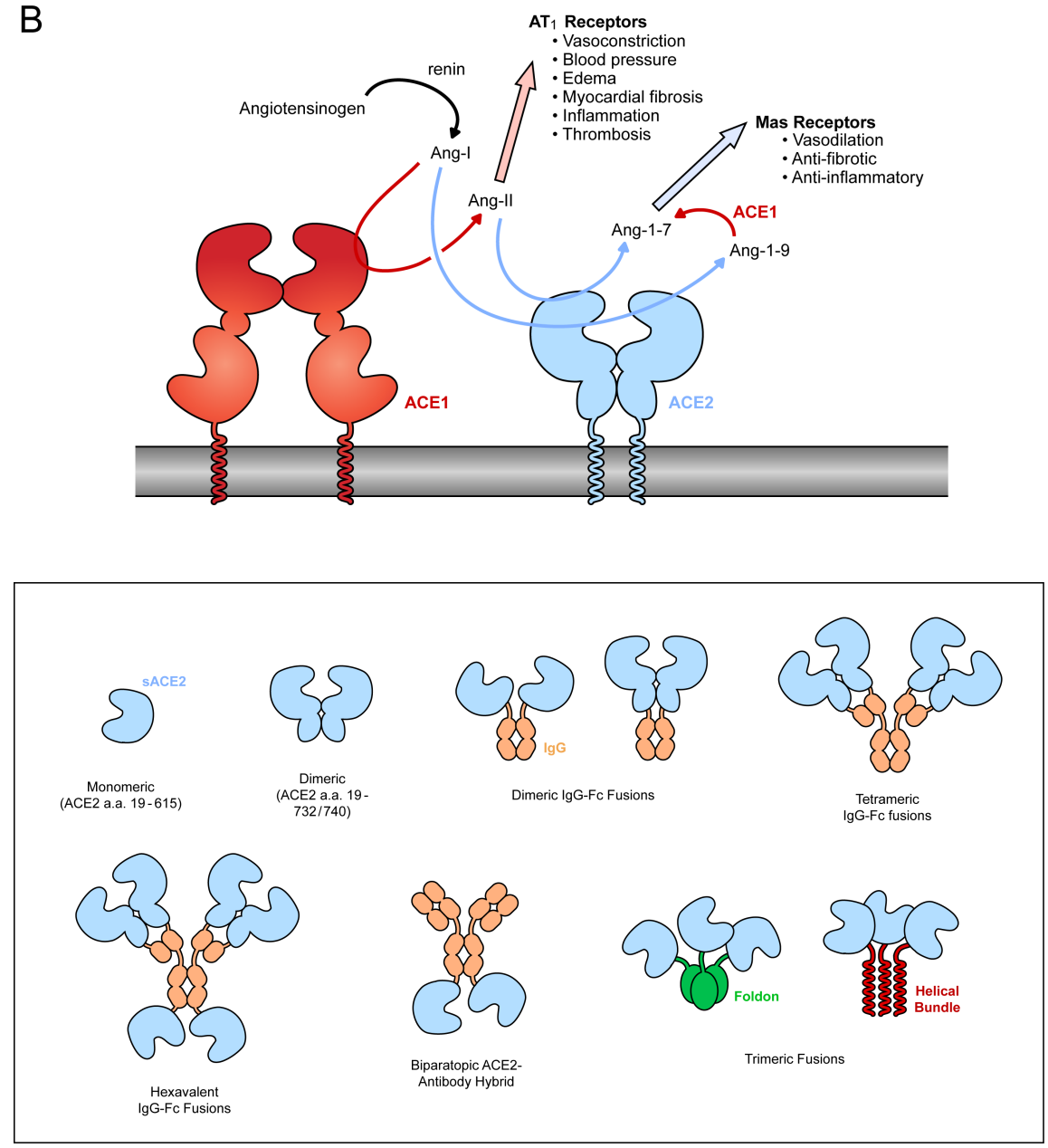